\title{
Research and Design of Operation and Maintenance of Digital Factory System Based on VR IAR Technology
}

\author{
Jiqing $\mathrm{CaO}^{1, \text { a }}$, Shuhai Zhang ${ }^{2, \mathrm{~b}}$ \\ ${ }^{1}$ Department of Information Engineering, Suzhou Industrial Park Institute of Service Outsourcing \\ ${ }^{22}$ Department of IT Service, Bosch Automotive Products (Suzhou) Co., Ltd. \\ aemail:grinner@tom.com, bemail:zhangsh @tom.com
}

Keywords: Virtual Reality; Augmented Reality; Digital Factory; Cloud Platform; Operation and Maintenance

\begin{abstract}
The existing industrial control user interface technology lacks of ability of expression and interaction in the performance of digital factory automation and large-scale complex equipment operating state. This paper designs the Operation and Maintenance architecture of the digital factory system based on Virtual Reality and Augmented Reality technology, expands the control end to more friendly 3D virtual reality interface, and realizes the interoperability integration of the heterogeneous remote equipment and system together with the technology of RFID, OPC protocol and ESB API. The designed Operation and Maintenance system is built on the cloud platform, including the Layer of Data Acquisition, Data Processing and Data Presentation and achieves the common Operation and Maintenance functions such as Data Collection, Equipment Maintenance, Incident Management, Spare Parts Management and Staff Training etc. The system has good openness, adaptability and scalability and the prototype system has been implemented in the joint venture, and achieved good result.
\end{abstract}

\section{Introduction}

Digital factory is an important foundation for the realization of Industry 4.0. Based on the data of the whole life cycle of the product, digital factory applies the virtual simulation technology to simulate, analyze, evaluate, validate and optimize different aspects of planning, manufacturing and operation. Digital factory is characterized by a large number of equipment and complex system environment, so the equipment failure is caused by a variety of reasons, which puts forward higher demand for the timely maintenance of the equipment of the digital factory [1]. The current user interface (UI) and maintenance manual of industrial control system is a typical two-dimensional graphical user interface, whose expression and interaction are inadequate and inconvenience, so that they have limited guidance on the maintenance of equipment.

As one of the core standards of Industry 4.0, digital twins make Virtual Reality (VR) technology become the latest development direction of human-computer interaction. Based on Virtual Reality and Augmented Reality (VR/AR) technology to build intelligent virtual environment can enhance the existing industrial control interface environment effectively, increase the dimension of information interaction and intelligence to help users closer to the physical reality, improve data analysis speed and accuracy, which is great important to the system maintenance of the digital factory. [2]

This paper is to explore and practice the application of VR/AR technology in the operation and maintenance (O\&M) of digital factory system, and realize the synchronization between the VR model and the physical state through the technologies of RFID, sensor system and OPC protocol etc. By applying technologies of VR/AR, Big Data and intelligent positioning to the virtual scene and the real environment, it not only provides the design capability, but also can realize the new application value of the O\&M of the digital factory system. 


\section{Digital Factory and VR/AR}

Supported by a high-performance computer and high-speed network, the digital factory uses technologies of computer simulation, virtual reality and process automation to work together to achieve the functions of design, manufacturing, operation, quality control of the product life cycle. Digital factory realizes the function of digital twins of Industry 4.0, completes the mapping in the VR space, which reflects the corresponding physical equipment life cycle process, and ultimately creates efficient and flexible production.

VR is a new human-computer interface communication method, which provides users with a presence and multi-sensory channel experience. VR system can be divided into three categories, i.e., desktop VR system, immersive VR system and distributed VR system. Desktop VR uses standard CRT (Cathode Ray Tube) display and stereoscopic display technology, usually used in engineering CAD (Computer Aided Design) and other applications. The immersive VR system uses a wearable device to enclose the user's vision, hearing, and other sensations, creating an illusion of being in a virtual environment. Based on the immersion VR system, distributed VR system connects the different users together to share the same virtual space through the network, so that get the user experience to a higher realm.

Augmented Reality (AR) is an effort to enhance the understanding and interaction of real-world environments by integrating the virtual environment generated by the computer with the real environment. In the O\&M scenario, engineers can assemble or repair equipment through the guidance of wearable device such as AR glasses, and locate the equipment and the location of spare parts in the distribution. It can also achieve the function that the screen-side headquarters guide the on-site O\&M engineers to repair equipment supported by AR wearable device. [3]

Digital factory has complex system environment and a wide range of equipment, the introduction of VR/AR technology can achieve the visualization of the O\&M of the equipment and the personnel training, which improves human-computer interaction and enhance the presence of people. Digital factory technology has changed the concept of traditional system O\&M, realized the computer-aided digital simulation and optimization of accurate and reliable automated operation from the experience and manual methods, which has important significance in time, quality and cost aspects of the system O\&M.

\section{System Architecture Design}

\subsection{Design Guidelines and Objectives}

The design goal of this system is to construct a virtual O\&M platform with high telepresence and interactivity for the digital factory system by means of technologies of VR/AR and cloud platform etc. According to the actual needs of the digital factory and the characteristics of the VR system, in the process of constructing the system O\&M platform, the following design criteria are mainly followed: [4]

(1) Openness. It should ensure that the system can be compatible with existing different systems, hardware and network equipment, and easy to upgrade and improve the system later.

(2) Distributed. The scope of the digital factory system involves different directions of the integrated system, including horizontal, vertical and end to end systems, so that the O\&M system must be an information integration framework to support distributed and heterogeneous systems.

(3) Interactivity. To establish the digital twins for the physical system and virtual system of the digital factory, and achieve the interactive O\&M functions of the physical system in the virtual system.

3.2 Technical Architecture Design

Based on the above design requirements, this paper designs a technical architecture of a general digital factory O\&M system based on technologies of VR/AR, OPC (OLE for Process Control), ESB (Enterprise Service Bus) and cloud computing (see Figure 1) etc. The real-time O\&M of the 
field equipment can be realized by real-time updating the data of the field control devices. Display\& Interaction Layer App\& Service Layer Acquisition\& Control Layer

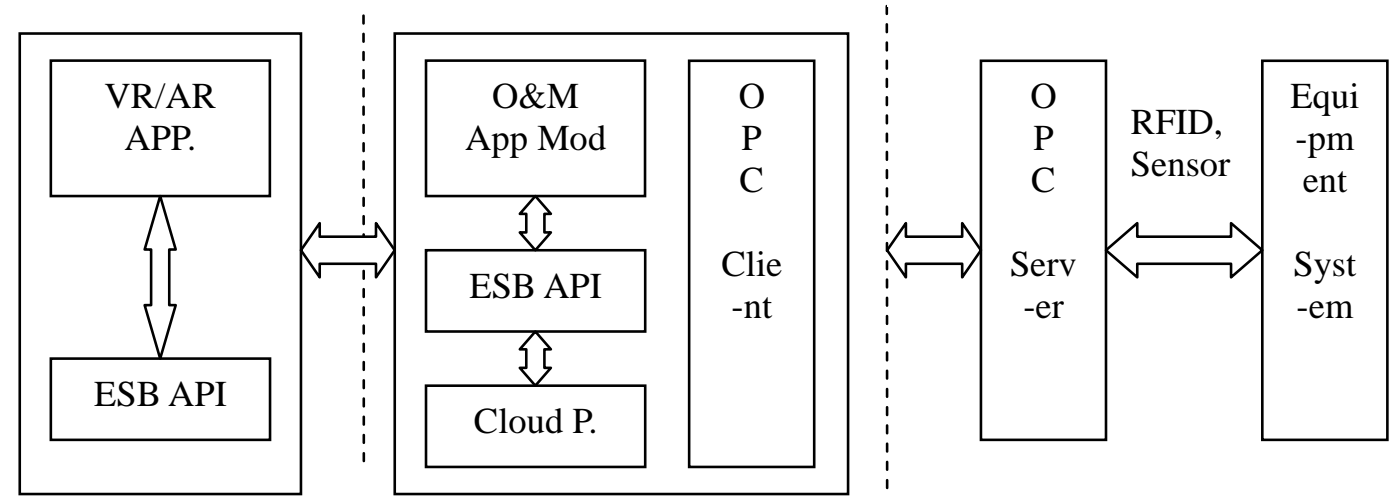

Fig. 1- Digital Factory O\&M System Technical Architecture

The technical architecture of the system adopts three layers, namely, Acquisition and Control Layer, Application and Service Layer and Display and Interaction Layer. The layers are relatively independent and are interrelated in a request / service manner. The Acquisition and Control Layer provides application service in the form of OPC server. With RFID (Radio Frequency Identification) and sensing system, the data acquisition and real-time control of different equipment on site can be realized. [5] OPC defines the method of exchanging real-time data automatically between different devices, which has become a universal data communication interface, and has been widely supported by industrial hardware manufacturers. [6] OPC server and the cloud-based Application and Service Layer constitute the local data source server of the distributed system. The Application and Service Layer is the communication bridge between the on-site OPC server and the remote $\mathrm{VR} / \mathrm{AR}$ system. On the one hand, it realizes the function of OPC client based on the standard OPC interface and OPC Server, on the other hand, it stores and processes relevant data, realizes the intercommunication between VR/AR system and other remote and heterogeneous systems through ESB API technology. [7]

\subsection{Functional Architecture Design}

From the perspective of logic function, the system architecture is designed in Figure 2. The architecture is divided into three layers, namely, Data Layer, Service Layer and Interactive Interface Layer, in which the Data Layer and Service Layer are built in the SaaS (Software as a Service) layer on the cloud platform, the Interactive Interface Layer is the remote client. The Interactive Interface Layer is a unified interface between user and O\&M system. Through VR/AR technology, the function of display and human-computer interaction such as Visual Management of Equipment, Spare Parts, Incident Alarm and Positioning and Personnel Training are realized. The Service Layer implements the back-end functions of the Interactive Interface Layer, including Equipment Accounting Management, Equipment Maintenance Management, Spare Parts Management, Incident Management, O\&M Personnel Training and Environment Modeling. The Data Layer mainly realizes the data storage functions such as Equipment Information Base, Spare Part Storehouse, O\&M Knowledge Base and VR Model Base. Specific functions are described below: 


\begin{tabular}{|l|c|}
\hline $\begin{array}{l}\text { Interactive } \\
\text { Interface } \\
\text { Layer }\end{array}$ & $\begin{array}{c}\text { Visual Mgt of Equipment, Spare } \\
\text { Parts, Incident and Positioning, } \\
\text { Personnel Training }\end{array}$ \\
\hline $\begin{array}{l}\text { Service } \\
\text { Layer }\end{array}$ & $\begin{array}{c}\text { Mgt of Equipment Account, } \\
\text { Equipment Maintenance, Spare } \\
\text { Parts, Incident, Training etc }\end{array}$ \\
\hline Data Layer & $\begin{array}{c}\text { Information Base of Equipment, } \\
\text { Spare Part, O\&M Knowledge and } \\
\text { VR Model etc }\end{array}$ \\
\hline
\end{tabular}

Fig. 2- Digital Factory O\&M System Functional Architecture

(1) Equipment Account Management

Equipment Account Management is the basis of equipment maintenance management, which storages the basic information of the equipment and provides unified data base information for other modules. This module realizes the maintenance of the basic information of the equipment and their accessory parts, and the three-dimensional VR graphics so that the text and graphics can be combined to truly realize the visualization of equipment management.

(2) Equipment Maintenance Management

This module realizes the O\&M management of the equipment, whose purpose is to ensure that the equipment is in a good state of technology to meet the needs of production operations, and make the costs of the maintenance the most economical. This module implements the following functions:

(a) Maintenance information is automatically acquired

With technologies of AR wearable devices and RFID, the system can automatically know the product model and maintenance records, show the specific composition of the production line and equipment, or even how to disassemble the parts, which can greatly reduce maintenance time.

(b) Provides a unified management of the O\&M work orders

Users can implement visual maintenance works like maintaining, repairing, point inspection and incident reporting etc for equipment.

(c) Realization of virtual disassembling simulation

Disassembly and assembly of key equipment is an important work in the repair process. The correct disassembly process will have a significant impact on the effectiveness of equipment overhaul and staff training. Three-dimensional VR has a strong field practicality to help the designers and field experts maximize the work efficiency. [8]

(3) Spare Parts Management

Working with RFID technology, the module achieves automatic data acquisition and positioning of spare parts, and management of the warehousing, storage and inventory of spare parts. When the number of spare parts inventory is not enough, it can carry out early warning, automatically generate the purchase orders, and timely replenish the lack of the amount.

(4) Incident Management

(a) Incident Alarm and Processing

This module realizes the functions of incident discovery, processing and the spare part replacement. When the real-time data of the collected field equipment is processed by the diagnosis system, the system will display the alarm signal on the VR UI and a hyperlink will appear in the corresponding equipment location. The operator can click the link to enter the equipment incident of the virtual scene display. In the same time, the system can make the best maintenance program, including maintenance priority, technology program, owner, scheduling, spare parts, preparation work, tools and acceptance methods and standards with the supporting of the of historical maintenance data. [9] 
(b) Incident Early Warning and Prevention

This module is based on the Big Data technology, it can predict the state of the system and make corresponding maintenance decision by analyzing the main parameters and indexes representing the performance of the system and equipment, which can effectively prevent the occurrence or expansion of failure and reduce the economic losses caused by failure. [10]

(5) Training Management

Utilizing the advantages of VR/AR technology, the trainee can control the operation of the device through his or her limbs, mouse and keyboard, and display the operation status and related information of the various parts in the O\&M scene, so that the trainee has a real feeling of the scene which will greatly enhance the learning effect. [11]

\section{(6) O\&M Environment Modeling}

The three-dimensional O\&M environment of the digital factory includes hardware environment and software environment. Hardware environment modeling mainly refers to the establishment of models for the manufacturing environment, including the workshop, production lines, console and equipment. Software environment modeling mainly refers to the implementation of the functions of production line, equipment assembly and maintenance, process and workshop roaming. O\&M staffs can intuitively understand the layout, composition and association of factories, workshops, production lines and equipment, as well as the production process through the VR system.

\section{Implementation of the Design}

The O\&M platform based on VR/AR technology designed in this paper has been implemented as prototype system and been tested in MOTO Auto Parts (Suzhou) Digital Factory System. In the prototype system, the three-dimensional modeling of the assembly workshop is completed, and the centralized data acquisition and monitoring of the remote and heterogeneous physical devices is realized. The core visual O\&M functions are realized in the remote client. After the O\&M prototype system is put into use, it has achieved the expected effect. The O\&M function has met the requirements of the O\&M staff, and assisted the staff to complete the O\&M of the equipment quickly and effectively. At present, the prototype system works well and has verified the feasibility and validity of the design of the system. It is an effective exploration of the application of VR/AR technology in the field of digital factory system O\&M.

\section{Summary}

To meet the requirement of digital factory and the limitations of current O\&M, this paper designs cloud-based O\&M system architecture based on VR/AR, and realizes the user interface which is more friendly and interactive. It realizes the real-time gathering of the equipment data based on the technologies of RFID and the sensor system. It realizes the unified management of the remote, heterogeneous system environment based on OPC and the ESB API technologies, which provides a practical solution to the digital factory O\&M system. The designed architecture of the O\&M system has been realized as a prototype system in the joint venture, and achieved good results. However, further improvements will be needed in the future study, such as collaboration and integration with other subsystems of the cloud-based O/M system, such as Big Data, Intelligent Positioning and Artificial Intelligence etc.

\section{Acknowledgement}

In this paper, the research was sponsored by the Service Outsourcing Foundation of Suzhou Institute Park Service Outsourcing Institute (Project No. ky-xjy02).

\section{References}

[1] Tao Liyan, Wang Zhifeng, Nie Qing, Liang Mantang. Study on Workshop Layout and 
Production Line Simulation for Digital Factory [J]. Journal of Hangzhou Dianzi University, 2014, (6)

[2] Du Yi, Song Guanghong. Construction of Digital Factory of Oil and Gas Field Based on Virtual Reality Technology[J]. Oil-Gasfield Surface Engineering, 2015(8)

[3] RAN Yang, ZHU Fei, CHEN Kang. Virtual and Augmented Reality Applications in Industrial Design[J]. LASER JOURNAL, 2010, 31(1)

[4] Zhang Qian, Qi Deyu. Service-Oriented Collaborative Design Platform for Cloud Manufacturing [J]. Journal of South China University of Technology (Natural Science Edition), 2011, 39(12)

[5] Zhang Weikun, Zhang Qiao, Lin Zhiwen. Technology of Instrument Communication and Test Data Collection Control Based on RFID [J]. Computer Measurement \& Control, 2015, 23(12)

[6] WANG Shiwen, ZHANG Ning, SHEN Zhipeng, GUO Chen, WANG Guo-feng. OPC technology based data acquisition and monitoring system for marine engine room[J], Journal of Dalian Maritime University,2014, (3)

[7] Guo Zhi-qiang. Analysis and Design of Data Communication of Heterogeneous Systems in Cloud Computing Environment[J]. Information Security and Technology, 2016, 7(2)

[8]Xiang Maoying, Ma Chunyong, Han Yong, Huo Peng, Wang Chun. Research on Chemical Equipment Virtual Training System Based on Unity3D [J]. Computer Technology and Development, 2014, (7)

[9] DENG Li, LU Chenyao, SHAO Yuying, LU Gen, FEI Minrui. Application of Augmented Reality Technology in Incident Source Identification of Power System Equipment [J]. Journal of System Simulation, 2014, 26(10).

[10] Liang Dongsheng, Yin Xunzheng, Sun Wang. Research on Active Maintenance System of Metro Signal Equipment Based on Large Data Processing [J]. Chinese Railways, 2014, (10)

[11] HU Hong, CHAO Jiangang, LIN Wanhong, XIONG Ying, YANG Jin. Astronaut Virtual Assembly Training Simulation Based on Virtual Hand Interaction [J], Journal of Computer Applications,2015, (z2) 